Nathan Cade, MD; Brad Granath, MD Jon O. Neher, MD Valley Family Medicine Residency, University of Washington at Valley, Renton

Sarah Safranek, MLIS Health Sciences Librarian Emeritus, University of Washington Medical School, Seattle

DEPUTY EDITOR Gary Kelsberg, MD Valley Family Medicine Residency, University of Washington at Valley, Renton

doi: $10.12788 / \mathrm{jfp} .0231$

\title{
Q/Can family physicians accurately screen for AAA with point-of-care ultrasound?
}

\author{
EVIDENCE-BASED ANSWER
}

$\mathrm{A}$

LIKELY YES. Point-of-care ultrasound (POCUS) screening for abdominal aortic aneurysm (AAA) by nonradiologist physicians is $98 \%$ sensitive and $99 \%$ specific, compared with imaging performed by radiologists (strength of recommendation

\section{Evidence summary}

Meta-analysis demonstrates accuracy of nonradiologist providers with POCUS

A systematic review and meta-analysis (11 studies; 946 exams) compared nonradiologist-performed AAA screening with POCUS vs radiologist-performed aortic imaging as a gold standard. Eight trials involved emergency medicine physicians (718 exams); 1 trial, surgical residents (104 exams); 1 trial, primary care internal medicine physicians (79 exams); and 1 trial, rural family physicians (45 exams). The majority of studies were conducted in Ireland, the United Kingdom, Australia, and Canada, with 4 trials performed in the United States. ${ }^{1}$

Researchers compared all POCUS exam findings with radiologist-performed imaging (using ultrasound, computed tomography, magnetic resonance imaging, or angiography) and with operative findings or pathology where available. There were 193 true positives, 8 false-positives, 740 true negatives, and 5 false-negatives. Primary care physicians identified 6 patients with AAA, with no false-positives or false-negatives. Overall, POCUS demonstrated a sensitivity of 0.975 ( $95 \%$ CI, 0.942 to 0.992 ) and a specificity of 0.989 (95\% CI, 0.979 to 0.995$)$. ${ }^{1}$

Nonradiologist providers received POCUS
[SOR]: B, meta-analysis of diagnostic accuracy studies mostly involving emergency medicine physicians). European family physicians demonstrated 100\% concordance with radiologist readings (SOR: C, very small subsequent diagnostic accuracy studies). training as follows: emergency medicine residents, 5 hours to 3 days; emergency medicine physicians, 4 to 24 hours of didactics, 50 AAA scans, or American College of Emergency Medicine certification; and primary care physicians, 2.3 hours or 50 AAA scans. Information on training for surgical residents was not supplied. The authors rated the studies for quality (10-14 points on the 14-point QUADA quality score) and heterogeneity $\left(I^{2}=0\right.$ for sensitivity and $I^{2}=.38$ for specificity). ${ }^{1}$

\section{European studies support FPs' ability to diagnose AAA with POCUS}

Two subsequent prospective diagnostic accuracy studies both found that POCUS performed by family physicians had $100 \%$ concordance with radiologist overread. The first study (in Spain) included 106 men (ages 50 and older; mean, 69 years) with chronic hypertension or a history of tobacco use. One family physician underwent training (duration not reported) by a radiologist, including experience measuring standard cross-sections of the aorta. Radiologists reviewed all POCUS images, which identified 6 patients with AAA (confirmed by CT scan). The concordance between the family physician and the radiologists was absolute (kappa = 1.0; sensitivity and specificity, 
$100 \%$; positive and negative predictive values, both 1.0). ${ }^{2}$

The second study (in Denmark) compared 29 POCUS screenings for AAA performed by 5 family physicians vs a gold standard of a radiologist-performed abdominal ultrasound blinded to previous ultrasound findings. Four of the family physicians were board certified and 1 was a final-year resident in training. They all underwent a 3-day ultrasonography course that included initial e-learning followed by 2 days of handson training; all passed a final certification exam. The family physicians identified 1 patient with AAA. Radiologists overread all the scans and found $100 \%$ agreement with the 1 positive AAA and the 28 negative scans. ${ }^{3}$

\section{Recommendations from others}

In 2019, the US Preventive Services Task Force (USPSTF) offered a Grade "B" (moderate net benefit) recommendation for screening with ultrasonography for AAA in men ages 65 to 75 years who have ever smoked, and a Grade "C" recommendation (small net benefit) for screening men ages 65 to 75 years who have never smoked. ${ }^{4}$ In 2017, the Canadian Task Force on Preventive Health Care recommended screening all men ages 65 to 80 years with 1 ultrasound exam for AAA (weak recommendation; moderate-quality evidence).
The Canadian Task Force also noted that, with adequate training, AAA screening could be performed in a family practice setting. ${ }^{5}$

\section{Editor's takeaway}

While these studies evaluating POCUS performed by nonradiologists included a small number of family physicians, their finding that all participants (attending physicians and residents) demonstrate high sensitivity and specificity for AAA detection with relatively limited training bodes well for more widespread use of the technology. Offering POCUS to detect AAAs in family physician offices has the potential to dramatically improve access to USPSTF-recommended screening. JFP
References
1. Concannon E, McHugh S, Healy DA, et al. Diagnostic accuracy of non-radiologist performed ultrasound for abdominal aortic aneurysm: systematic review and meta-analysis. Int J Clin Pract. 2014;9:1122-1129. doi: 10.1111/ijcp.12453
2. Sisó-Almirall A, Gilabert Solé R, Bru Saumell C, et al. Feasibility of hand-held-ultrasonography in the screening of abdominal aortic aneurysms and abdominal aortic atherosclerosis [article in Spanish]. Med Clin (Barc). 2013;141:417-422. doi: 10.1016/ j.medcli.2013.02.038
3. Lindgaard K, Riisgaard L. 'Validation of ultrasound examinations performed by general practitioners'. Scand J Prim Health Care. 2017;3:256-261. doi: 10.1080/02813432.2017.1358437
4. US Preventive Task Force. Screening for abdominal aortic aneu- rysm: US Preventive Services Task Force Recommendation State- ment. JAMA. 2019;322:2211-2218. doi:10.1001/jama.2019.18928
5. Canadian Task Force on Preventive Health Care. Recommenda- tions on screening for abdominal aortic aneurysm in primary care. CMAJ. 2017;189:E1137-E1145. doi: 10.1503/cmaj.170118

This supplement was sponsored by Boehringer Ingelheim Pharmaceuticals, Inc. It was edited and peer reviewed by The Journal of Family Practice.

\section{Type 2 Diabetes 2021}

The management of type 2 diabetes (T2D) has changed substantially over the last several decades. Standard practices have moved away from the glucocentric approach of T2D management into an era in which the interplay among T2D, obesity, atherosclerotic cardiovascular disease, heart failure, and chronic kidney disease is increasingly recognized.

This supplement to The Journal of Family Practice brings together key updates in the field of T2D to help physicians care for patients who have not only T2D, but other interrelated diseases.

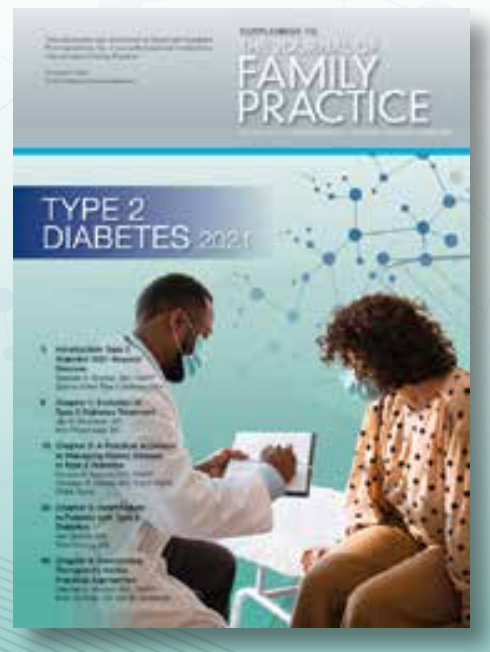

This supplement can be found at www.mdedge.com/T2D2021. 